s8.m28.p2 Influence of partial substitutions of bismuth for potassium and copper for lithium on structural and electric properties of $\mathrm{Bi}_{2} \mathrm{CuO}_{4}$ compound. Ahmed Bekka, Mayouf Sellami, Noureddine Bettahar, University of Science and Technology of Oran, Faculty of Chemistry, Nedjari Mohamed El-Makkari, 9, Oran, Algeria. E-mail:bekkahmed@yahoo.fr

\section{Keywords: $\mathrm{Bi}_{2} \mathrm{CuO}_{4}$; Ionic substitution; Electrical resistivity}

Poly-crystalline solid solutions with chemical formula $\mathbf{B i}_{2-\mathrm{x}} \mathbf{K}_{\mathrm{x}} \mathbf{C u}_{1-\mathrm{y}} \mathbf{L i}_{\mathrm{y}} \cdot \mathbf{O}_{4}$ derived from $\mathbf{B i}_{2} \mathbf{C u O}_{4}$ compound have been synthesised using solid reaction method at high temperature. The samples have been characterized by means of $\mathrm{X}$-Ray Diffraction as well as conductivity measurements at room temperature on sintered pellets . The first characterization method allowed us to display, on the one hand, that a limit of saturation in potassium is reached by the solid solution and, on the other hand, that the lithium introduced in small proportions allows to push back this limit in stabilizing the disturbed structure. The second characterisation method shows that the presence of the big cation $\mathrm{K}$, combined with that of the small cation $\mathrm{Li}$, allows to increase significantly the electrical conductivity of the obtained materials compared to $\mathbf{B i}_{2} \mathbf{C u O}_{4}$ known to be an insulating compound. s8.m28.p3 Structural Study of Thermal Stability of Nanocrystalline Iron and Copper Prepared by High-Pressure Torsion Deformation. V. Cherkaska, Z. Matej, R. Kuzel, J. Pesicka, J. Cízek, I. Procházka ${ }^{a}$, R. K. Islamgaliev ${ }^{b}$, ${ }^{a}$ Faculty of Mathematics and Physics, Charles University in Prague, Ke Karlovu 5, 12116 Praha 2, Czech Republic; ${ }^{b}$ Ufa State Aviation Technical University, Ufa, Russia E-mail: cherkas@karlov.mff.cuni.cz

Keywords: Nanocrystalline materials; Powder diffraction; Thermal stability

Thermal stability of fine microstructure of the selected samples of iron and copper with addition of different amount of $\mathrm{Al}_{2} \mathrm{O}_{3}$ prepared by high-pressure torsion deformation under 6 $\mathrm{GPa}$ is investigated by X-ray powder diffraction (PXRD), positron life-time spectroscopy (PL) and transmission electron microscopy (TEM). Conventional powder diffraction studies were carried out in order to analyse lattice parameters, texture coefficients and line broadening. In simplified analysis, the profiles were fitted with analytical functions and analyzed in terms of integral breadths. More sophisticated analysis was performed by total powder diffraction pattern fitting as proposed by Ungar et al [1] and Scardi \& Leoni [2]. The crystallite size in the order of 50-300 $\mathrm{nm}$ and dislocation densities of $1 \times 10^{14} \mathrm{~m}^{-2}$ to $1 \times 10^{15} \mathrm{~m}^{-2}$ were determined by all the methods. Typical line broadening anisotropy was evaluated by the dislocation orientation (contrast) factors. PL spectra shown two major components - one comes from positrons trapped at dislocations inside the distorted regions and another that can be attributed to positrons trapped in microvoids with the size of 4-5 vacancies. Obtained values of crystallite size and dislocation density agree quite well with the estimations made from TEM for $\mathrm{Cu}+0.5$ wt. $\%$ of $\mathrm{Al}_{2} \mathrm{O}_{3}$ and $\mathrm{Fe}$ samples. On some of the TEM pictures of the former sample the $\mathrm{Al}_{2} \mathrm{O}_{3}$ droplets were found. It was found that the addition of at least 0.5 wt. $\% \mathrm{Al}_{2} \mathrm{O}_{3}$ prevents grain growth and keeps the dislocation density high and crystallites small up to about $400{ }^{\circ} \mathrm{C}$. The amount of $0.3 \mathrm{wt}$. $\%$ is insufficient for that and rapid grain growth is observed at about $200{ }^{\circ} \mathrm{C}$. For iron samples continuous strain recovery is observed in the range $130-200{ }^{\circ} \mathrm{C}$. Valuable results were obtained by old and simple back-reflection method. For as-prepared samples, continuous rings were observed while after annealing a few dots were clearly distinguished on them indicating fast growth of only a few grains. This appeared at lower temperatures, before any significant changes of line broadening and PL spectra and it is more pronounced for copper samples. In-plane inhomogeneity was also detected by the technique. In margin regions of disc specimens, larger grains were detected than in their centers. With increasing annealing temperature such effects can be seen also on diffraction profiles. The bimodal crystallite size distribution is a typical structural feature during annealing of the HPT deformed metals.

The research is supported by the Grant Agency of the Czech Republic (106/02/1521)

[1] G.Ribárik,T.Ungár,J.Gubicza (2001).J.Appl.Cryst. 34, 669- 676.

[2] P. Scardi, M. Leoni, Y.H. Dong (2000). Eur. Phys. J. B 18, 23-30. 